\title{
Relação entre os tipos de aleitamento materno e o consumo de vitamina $A$ e ferro em crianças de 6 a 12 meses
}

\author{
Relationship between breastfeeding patterns and intake of vitamin \\ A and iron in children 6-12 months
}

\author{
Mariane Alves Silva (https://orcid.org/0000-0002-6518-6534) ${ }^{1}$ \\ Marcela Martins Soares (http://orcid.org/0000-0001-9920-2814) ${ }^{1}$ \\ Poliana Cristina de Almeida Fonseca (http://orcid.org/0000-0002-8875-5154) ${ }^{2}$ \\ Sarah Aparecida Vieira (https://orcid.org/0000-0002-0304-2711) ${ }^{1}$ \\ Carolina Abreu Carvalho (https://orcid.org/0000-0001-7900-4642) ${ }^{3}$ \\ Raquel Maria Amaral (http://orcid.org/0000-0003-1323-8707) ${ }^{1}$ \\ Sylvia do Carmo Castro Franceschini (https://orcid.org/0000-0001-7934-4858) ${ }^{1}$ \\ Juliana Farias de Novaes (https://orcid.org/0000-0003-3616-5096) ${ }^{1}$
}

${ }^{1}$ Universidade Federal de Viçosa (UFV). Av. Peter Rolfs s/no, Campus Universitário 36570-900

Viçosa MG Brasil. mariane.nutricaoufv@ gmail.com

${ }^{2}$ Universidade Federal do Piauí (UFPI). Teresina PI Brasil.

${ }^{3}$ Universidade Federal do

Maranhão (UFMA). São

Luís MA Brasil.

\begin{abstract}
The aim of this study was to analyze the association between breastfeeding type in the first six months of life and intake of vitamin $A$ and iron in children 6-12 months of age. It is a cohort study with 226 children. The type of breastfeeding from 1-6 months of life and the food intake from 6 to 12 months were evaluated. Nutrient intake between groups was compared by the Kruskal-Wallis test. The association between nutrient intake and type of breastfeeding was analyzed by Poisson Regression. Consumption below the recommendation of vitamin $A$ and iron was $33.6 \%$ and $67.7 \%$, respectively. Infants exclusively and predominantly breastfed from birth to two months had higher vitamin A intake from 6-12 months of life. Infants who were breastfeeding at 6 months had higher vitamin A intake and lower iron by the bivariate analysis. The group that received mixed feeding and artificial feeding at 1 month and the group non-breastfed at 6 months had vitamin A intake below the recommended from 6-12 month of life. The results reinforce the importance of exclusive breastfeeding in the first six months of life contributing to the higher vitamin A intake. Iron intake was lower among breastfed children, but the bioavailability of this nutrient in breast milk is higher.
\end{abstract}

Key words Breastfeeding, Vitamin A, Iron.
Resumo O objetivo deste artigo é analisar a associação entre o tipo de aleitamento no primeiro semestre de vida e o consumo de vitamina $A e$ ferro do $6^{\circ}$ ao $12^{\circ}$ mês. Estudo de coorte com 226 crianças. Avaliou-se o tipo de aleitamento do $1^{\circ}$ ao $6^{\circ}$ mês de vida e o consumo alimentar do $6^{\circ}$ ao $12^{\circ}$ mês. O consumo de nutrientes entre os grupos foi comparado pelo teste Kruskal-Wallis. A análise da associação entre o consumo de nutrientes e o tipo de aleitamento se deu pela Regressão de Poisson. O consumo abaixo da recomendação de vitamina A e ferro foi de 33,6\% e 67,7\%, respectivamente. Crianças amamentadas de maneira exclusiva e predominante no $1^{\circ}$ e $2^{\circ}$ mês tiveram maior ingestão de vitamina $A$ do $6^{\circ}$ ao $12^{\circ}$ mês de vida. Já as que estavam em aleitamento materno no $6^{\circ}$ mês tiveram maior ingestão de vitamina $A e$ menor de ferro na análise bivariada. O grupo que recebeu aleitamento materno misto e artificial no $1^{\circ}$ mês e os não amamentados no $6^{\circ}$ mês tiveram consumo de vitamina $A$ abaixo do recomendado do $6^{\circ}$ ao $12^{\circ}$ mês de vida. Os resultados reforçam a importância do aleitamento materno exclusivo nos seis primeiros meses de vida, contribuindo para o maior consumo de vitamina A. O consumo de ferro foi menor entre as crianças amamentadas, porém, a biodisponibilidade desse nutriente no leite materno é maior.

Palavras-chave Aleitamento Materno, Vitamina A, Ferro. 


\section{Introdução}

A alimentação da criança, desde o nascimento até o primeiro ano de idade, exerce influência em sua saúde ao longo da vida ${ }^{1}$. Sabendo que o aleitamento materno é essencial para a alimentação infantil, a Organização Mundial da Saúde (OMS) recomenda o aleitamento materno exclusivo (AME) até os seis primeiros meses e o complementar até dois anos de idade ${ }^{2,3}$.

A introdução precoce da alimentação complementar pode resultar em menor duração do aleitamento materno e má absorção de alguns nutrientes, como a vitamina A e o ferro, levando ao desenvolvimento de carências nutricionais ${ }^{4-6}$. Em crianças, as deficiências de ferro e vitamina A constituem-se um problema de saúde públi$\mathrm{Ca}^{7,8}$. Dados da Pesquisa Nacional de Demografia e Saúde (PNDS) apresentam que a prevalência de anemia ferropriva e deficiência de vitamina A entre as crianças menores de cinco anos foi de $20,9 \%$ e $17,4 \%$, respectivamente ${ }^{9}$.

A associação entre o aleitamento materno nos seis primeiros meses de vida e a formação de hábitos alimentares saudáveis na infância ainda é controversa. Estudos apontam que a amamentação exerce influência direta na aceitação de alimentos em fases posteriores da vida ${ }^{10,11}$, indicando que crianças que foram amamentadas apresentam maior aceitação de frutas e legumes ${ }^{12}$. Acredita-se que essa prática tenha influência na ingestão de ferro e vitamina A após a introdução da alimentação complementar ${ }^{13}$. Entretanto, Valmórbida e Vitolo ${ }^{14}$ não mostraram associação entre a amamentação e práticas alimentares posteriores na infância. Sendo assim, o objetivo deste trabalho foi avaliar se o aleitamento materno no primeiro semestre de vida está relacionado à ingestão de vitamina A e ferro nas crianças com 6 a 12 meses de idade.

\section{Metodologia}

Trata-se de um estudo de coorte, realizado entre 2011 e 2013, com crianças acompanhadas até o primeiro ano de vida. $\mathrm{O}$ convite à participação no estudo foi realizado no único hospital-maternidade da cidade de Viçosa-MG, no momento do nascimento. A amostra foi de 226 crianças, de ambos os sexos, acompanhadas no setor de Imunização da Prefeitura Municipal de Viçosa-MG. Este serviço é considerado referência para realização do 'teste do pezinho', facilitando a captação das mães para o estudo.
O critério de inclusão foi o de a criança residir no município de Viçosa. Os critérios de exclusão foram: possuir doenças que alterassem o estado nutricional infantil; parto gemelar e consumo regular de medicamentos que alterassem a alimentação da criança. O fluxograma da amostra é apresentado na Figura 1.

As perdas de seguimento foram avaliadas quanto à possibilidade de viés de seleção até o sexto mês de acompanhamento. Não foram encontradas diferenças entre as crianças acompanhadas e perdidas, quando comparadas as suas características sociodemográficas, número de consultas pré-natal, variáveis de nascimento, como peso e comprimento ao nascer, e uso de chupetas, conforme apresentado nos trabalhos de Fonseca et al. ${ }^{15}$ e Carvalho et al. ${ }^{16}$.

Foi calculado um tamanho amostral para esse estudo no Programa OpenEpi considerando o total de crianças nascidas (806) em Viçosa durante a coleta de dados e a prevalência dos desfechos (consumo alimentar abaixo da recomendação para vitamina A - 33,3\% e ferro - 67,7\% no segundo semestre de vida das crianças). Para um nível de confiança de 95\%, o tamanho de amostra calculado foi de 237 e 249 considerando a prevalência de consumo alimentar abaixo da recomendação para ferro e vitamina $\mathrm{A}$, respectivamente. A amostra (226) de crianças de seis a doze meses acompanhadas desde o nascimento correspondeu a $91 \%$ do maior número amostral calculado (249 crianças).

Durante a entrevista, aplicou-se um questionário semiestruturado que continha questões referentes às condições de nascimento da criança e à situação socioeconômica. $O$ consumo do leite materno, fórmulas infantis e leite de vaca foi avaliado no primeiro, segundo, quarto e sexto mês, quando as crianças foram levadas à Policlínica para vacinação.

As mães ou responsáveis (com raras exceções o responsável) pelas crianças responderam a um recordatório de 24 horas no sexto, nono ou décimo segundo mês de vida. Após o primeiro semestre de vida, foi realizado apenas mais um encontro, no nono ou décimo segundo mês. Portanto, foi avaliado se existia diferença na ingestão alimentar de ferro e vitamina A das crianças no segundo semestre de vida, de acordo com o tipo de aleitamento materno que as mesmas apresentaram nos seis primeiros meses de vida. As perdas durante a avaliação do consumo alimentar ocorreram devido a algumas mães não saberem relatar o consumo das crianças, quando estas frequentavam creches em período integral e/ou ficavam com babás. 
Nasceram 806 crianças no período da coleta de dados

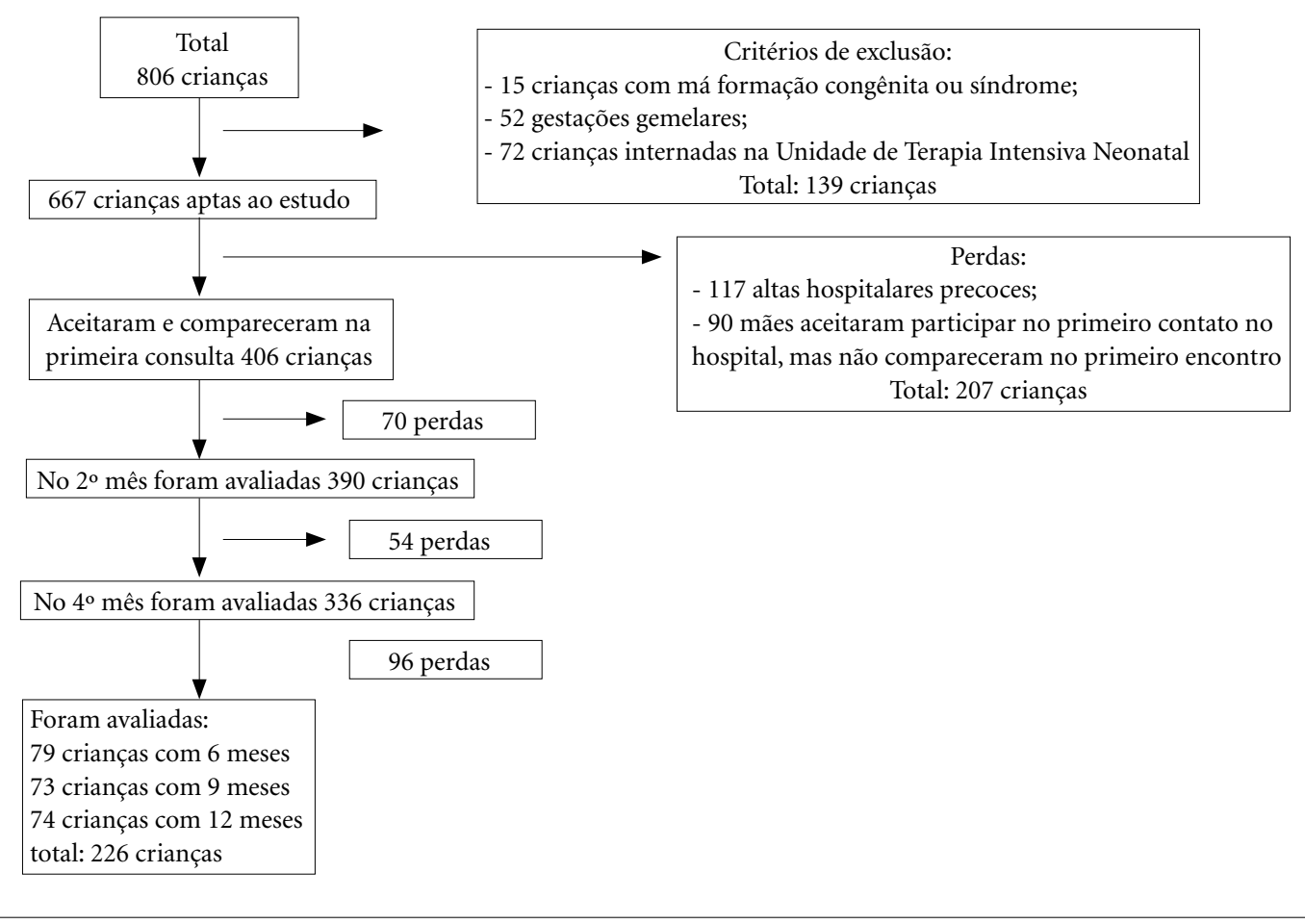

Figura 1. Fluxograma da amostra.

Para a classificação do tipo de aleitamento materno, utilizamos as definições adotadas pelo Ministério da Saúde ${ }^{3}$ : Aleitamento Materno Exclusivo (AME) - a criança recebe somente leite materno, direto da mama ou ordenhado, ou leite humano de outra fonte, sem outros líquidos ou sólidos, com exceção de xaropes, suplementos minerais ou medicamentos. Aleitamento Materno Predominante $(A M P)$ - além do leite materno é ofertado à criança água ou bebidas à base de água (chás, infusões) e sucos de frutas. Aleitamento Materno Complementado - a criança recebe, além do leite materno, alimento sólido ou semi-sólido com a finalidade de complementar o aleitamento, e não de substituí-lo. Aleitamento Materno Misto $(A M M)$ - a criança recebe leite materno e outros tipos de leite. Devido à baixa prevalência de AMP, consideramos os valores de ingestão desta categoria juntamente com o AME.

Os dados do consumo alimentar foram tabulados no software Avanutri ${ }^{\circledR}$. Foi selecionada a Tabela de Composição dos Alimentos (TACO $)^{17}$, $4^{\circ}$ versão. Os alimentos não encontrados no pro- grama foram cadastrados padronizando-se a lista de ingredientes, a fim de não subestimar ou superestimar a quantidade de vitamina $\mathrm{A}$ e ferro ingerida. A ingestão dos nutrientes foi comparada às Dietary Reference Intakes (DRI) ${ }^{18}$. A vitamina A foi quantificada em Equivalente de Retinol (RE) pelo software, porém, foi convertida em microgramas, para fins de análise.

Calculou-se a prevalência de inadequação do consumo de ferro utilizando a AI (Adequate Intake) para as crianças de 6 meses $(\mathrm{AI}=0,27 \mathrm{mg})$ e EAR (Estimated Average Requirement) para as crianças de 7 a 12 meses (EAR =6,9 mg). Quanto à vitamina $\mathrm{A}$, considerou-se $\mathrm{AI}=400 \mu \mathrm{g}$ para as crianças de 6 meses e $\mathrm{AI}=500 \mu \mathrm{g}$ para as criança de 7 a 12 meses. Utilizou-se a EAR como referência e, quando esta não era disponível, considerou-se a AI. Os nutrientes foram ajustados pela densidade de energia da dieta, pelo método de resíduos ${ }^{19}$. Esse método busca estimar a fração dos nutrientes que não se correlacionam com a ingestão total de energia ${ }^{20}$. Foi considerada a ingestão de ferro e vitamina A proveniente da dieta, 
não sendo contabilizado o consumo de suplementos alimentares, pois essa informação não constou nos recordatórios analisados.

As crianças que estavam em aleitamento materno no momento da aplicação do recordatório alimentar tiveram a quantidade de leite materno consumido estimada, a fim de se evitar a subestimação da ingestão alimentar. Para essa estimativa adotou-se a metodologia proposta pela $\mathrm{WHO} /$ $\operatorname{UNICEF}(1995)^{21}$.

A análise descritiva dos dados foi realizada pelas medidas de tendência central e de dispersão. Comparações entre os tipos de aleitamento materno e o consumo de nutrientes foram analisadas pelo teste Kruskal-Wallis. A análise do consumo de leite materno no sexto mês foi realizada pelo teste T de Student.

$\mathrm{Na}$ análise bivariada estimou-se o Risco Relativo (RR) e intervalo de confiança pela Regressão de Poisson com variância robusta. As variáveis que apresentaram valor de $\mathrm{p}<0,20$ foram consideradas para inclusão no modelo múltiplo. $\mathrm{Na}$ análise de regressão, a variável consumo alimentar foi ajustada pelo aleitamento atual, idade da criança, cor da criança, escolaridade da mãe e renda familiar. Para todas as análises foi utilizado o software Stata versão 12.0 e a significância estatística considerada foi $\alpha=0,05$.

O estudo foi aprovado pelo Comitê de Ética em Pesquisa com Seres Humanos da Universidade Federal de Viçosa, e financiado pela Fundação de Amparo à Pesquisa do Estado de Minas Gerais.

\section{Resultados}

Entre as crianças avaliadas $(n=226), 50,4 \%$ eram do sexo masculino, $35,0 \%$ com seis meses de vida, $32,3 \%$ com nove meses e $32,7 \%$ com um ano de idade. Das crianças avaliadas, 33,6\% tiveram consumo abaixo da recomendação de vitamina $\mathrm{A}$, enquanto que $67,7 \%$ de ferro. As mães das crianças possuíam idade média de 26 anos, e a maioria tinha mais de nove anos de estudo $(65,0 \%)$. Em relação à classificação socioeconômica da ABEP, 78,3\% das famílias encontravamse na categoria $\mathrm{C}, \mathrm{D}$ e $\mathrm{E}$.

Das crianças avaliadas, $77,9 \%$ foram amamentadas de maneira exclusiva ou predominante no primeiro mês de vida. Estas crianças apresentaram maior consumo mediano de vitamina $\mathrm{A}$ no segundo semestre de vida. A mediana de consumo de ferro entre as crianças que foram amamentadas de maneira exclusiva ou predominante no primeiro mês foi menor do que entre as que estavam em aleitamento artificial (Tabela 1).
O tipo de aleitamento que a criança apresentou no quarto mês de vida não interferiu na ingestão de ferro e vitamina A no segundo semestre de vida (Tabela 2). Crianças em aleitamento materno no sexto mês de vida apresentaram maior ingestão mediana de vitamina A e menor de ferro nesta idade $(\mathrm{p}<0,001)$ (Tabela 3$)$.

$\mathrm{Na}$ análise múltipla, observou-se que crianças que estavam em AMM (RR:1,65; IC95\%: 1,07 - 2,60) e AA (RR: 1,97; IC95\%: 1,10 - 3,58) no primeiro mês de vida, enquanto que as não amamentadas no sexto mês (RR: 1,81; IC95\%:1,10 $2,99)$ apresentaram maior risco de consumo de vitamina $\mathrm{A}$, abaixo da AI. Para o ferro, nenhuma associação estatisticamente significativa foi encontrada de acordo com o tipo do aleitamento (Tabela 4).

\section{Discussão}

Nesse estudo avaliou-se a influência do aleitamento materno nos primeiros seis meses de vida sobre o consumo de vitamina A e ferro no segundo semestre de vida das crianças. Verificou-se que aquelas que amamentaram de maneira exclusiva ou predominante no primeiro mês de vida tiveram maior consumo de vitamina A e menor consumo de ferro após introdução da alimentação complementar. No entanto, não foi encontrada associação entre o tipo de aleitamento no sexto mês e a ingestão de ferro no segundo semestre de vida.

Mais de um terço das crianças apresentaram consumo de vitamina A e ferro abaixo do recomendado. Esta prevalência foi inferior ao estudo de Fidelis e Osório ${ }^{22}$, o qual encontrou consumo de vitamina A abaixo do recomendado em $44 \%$ das crianças avaliadas na cidade de Pernambuco e 59,5\% em cidades da Região Metropolitana de Recife. Nesse mesmo estudo, 65\% das crianças apresentaram ingestão inadequada de ferro em Pernambuco, ao passo que na Região Metropolitana de Recife o resultado foi de 38,1\% de inadequação. No estudo de Freiberg et al. ${ }^{23}$, realizado em São Paulo, encontrou-se entre as crianças de 6 a 12 meses uma prevalência de adequação da vitamina A de $29,5 \%$ e de $52,2 \%$ em relação à ingestão de ferro.

Neste estudo, as crianças que estavam em aleitamento materno misto, artificial e não amamentaram no $6^{\circ}$ mês de vida apresentaram maior risco do consumo de vitamina $A$ abaixo da AI dos 6 aos 12 meses de vida. Kurihayashi et al. ${ }^{24}$ encontraram associação positiva entre tempo de aleitamento materno e concentrações séricas 
Tabela 1. Valores de mediana e intervalo interquartílico do consumo de nutrientes no segundo semestre de vida, de acordo com o tipo de aleitamento materno oferecido às crianças no primeiro e segundo mês. Viçosa-MG.

\begin{tabular}{|c|c|c|c|}
\hline Variáveis & $\begin{array}{c}\text { AME + AMP } \\
(n=176)\end{array}$ & $\begin{array}{l}\text { AMM } \\
(n=40)\end{array}$ & $\begin{array}{c}\text { AA } \\
(n=10)\end{array}$ \\
\hline \multicolumn{4}{|c|}{ Primeiro mês } \\
\hline Energia (Kcal) & $884,5(759,3-1068,1) \mathrm{a}$ & $962,5(808,6-1245,6) b$ & $903,1(793,5-1276,5) \mathrm{ab}$ \\
\hline Vitamina A (RE) & $611,1(443,5-880,0) \mathrm{a}$ & $513,1(253,8-778,6) b$ & $265,8(72,0-2010,5) \mathrm{ab}$ \\
\hline Ferro $(\mathrm{mg})$ & $4,5(2,8-6,8) \mathrm{a}$ & $5,0(2,7-9,0) \mathrm{ab}$ & $8,1(5,8-9,8) b$ \\
\hline \multicolumn{4}{|c|}{ Segundo mês } \\
\hline & $(n=160)$ & $(n=40)$ & $(n=16)$ \\
\hline Energia (Kcal) & $864.3(737.9-1063.3) \mathrm{a}$ & $986,5(861,7-1216,5) b$ & $953,7(811,1-1081,6) \mathrm{ab}$ \\
\hline Vitamina A (RE) & $605.6(453.3-813.4) \mathrm{a}$ & $584.7(258,6-1049,8) \mathrm{a}$ & $226,2(75,5-749,6) b$ \\
\hline Ferro $(\mathrm{mg})$ & $4,6(2,7-7,4)$ & $5,8(2,7-9,3)$ & $4,9(3,3-6,7)$ \\
\hline
\end{tabular}

AME: Aleitamento Materno Exclusivo; AMP: Aleitamento Materno Predominante; AMM: Aleitamento Materno Misto; AA: Aleitamento Artificial.

Letras diferentes representam significância estatística $(\mathrm{P}<0,05)$. Teste de Kruskal-Wallis

Tabela 2. Valores de mediana e intervalo interquartílico do consumo de nutrientes no segundo semestre de vida, de acordo com o tipo de aleitamento materno no quarto mês. Viçosa-MG.

\begin{tabular}{lcccc}
\hline Variáveis & $\begin{array}{c}\text { AME + AMP } \\
(\mathbf{n}=\mathbf{1 0 7})\end{array}$ & $\begin{array}{c}\text { AMM } \\
(\mathbf{n = 4 3})\end{array}$ & $\begin{array}{c}\text { AA } \\
(\mathbf{n}=\mathbf{1 9})\end{array}$ & $\begin{array}{c}\text { Aleitamento materno } \\
\text { complementado }(\mathbf{n}=\mathbf{3 7})\end{array}$ \\
\hline $\begin{array}{l}\text { Energia } \\
(\text { Kcal })\end{array}$ & $863,5(746,8-1031,4)$ & $912,8(786,6-127,2)$ & $973,5(934,0-1184,2)$ & $921,6(761,2-1092,5)$ \\
Vitamina & $603,5(437,4-814,5)$ & $597,6(275,9-810,5)$ & $489,0(101,5-153,9)$ & $575,7(314.5-812,3)$ \\
A (RE) & & a,b & & \\
$\begin{array}{l}\text { Ferro } \\
(\mathrm{mg})\end{array}$ & $4,6(2,6-7,5)$ & $5,4(3,2-7,6)$ & $4,9(1,9-7,9)$ & $4,5(2,7-6,6)$ \\
\hline
\end{tabular}

AME: Aleitamento Materno Exclusivo; AMP: Aleitamento Materno Predominante; AMM: Aleitamento Materno Misto; AA: Aleitamento Artificial.

Teste de Kruskal-Wallis. Letras diferentes representam significância estatística $(\mathrm{P}<0,05)$.

Tabela 3. Valores de mediana e intervalo interquartílico do consumo de nutrientes no segundo semestre de vida, de acordo com o aleitamento materno no sexto mês. Viçosa-MG.

\begin{tabular}{lccc}
\hline \multicolumn{1}{c}{ Variáveis } & $\begin{array}{c}\text { Sim }(\mathbf{n}=\mathbf{1 5 4}) \\
\text { Mediana }(\mathbf{p} 25-\mathbf{p 7 5})\end{array}$ & $\begin{array}{c}\text { Não }(\mathbf{n}=\mathbf{4 9}) \\
\text { Mediana }(\mathbf{p 2 5}-\mathbf{p 7 5})\end{array}$ & Valor de $\mathbf{p}$ \\
\hline Energia & $874,6(759,3-1026,1)$ & $973,5(793,5-1184,2)$ & 0,065 \\
Vitamina A (RE) & $654,7(492,0-941,4)$ & $401,9(101,5-673,2)$ & $<\mathbf{0 , 0 0 1}$ \\
Ferro (mg) & $4,3(2,6-6,0)$ & $8,9(5,8-10,4)$ & $<\mathbf{0 , 0 0 1}$ \\
\hline
\end{tabular}

Teste $\mathrm{T}$ de Student. Valores em negrito indicam significância estatística $(\mathrm{P}<0,05)$.

de retinol em crianças de 2 a 7 anos. As crianças que foram amamentadas exclusivamente por um período maior ou igual a 4 meses apresentavam maior concentração sérica de retinol ${ }^{24}$.

$\mathrm{O}$ grupo das frutas e verduras são as maiores fontes de vitamina $\mathrm{A}$, e alguns estudos indicam uma associação positiva entre a amamentação e o consumo desses grupos alimentares ${ }^{10-13}$. Foi identificado que o aleitamento materno exclusi- vo por maior tempo e, por consequência, o atraso do uso de fórmulas alimentares, foi associado a uma maior ingestão de vegetais aos cinco anos de idade ${ }^{25}$. Atribui-se a esse resultado a variedade de sabores que o leite materno possui. Assim, as crianças que são amamentadas reconhecem esses sabores em fases posteriores da vida e apresentam uma maior aceitação dos alimentos ${ }^{25,26}$. Além disso, espera-se que mães que se preocupem em 
Tabela 4. Análise de regressão múltipla dos tipos de aleitamento materno de acordo com o consumo abaixo da AI e da EAR de vitamina A e ferro, respectivamente, em crianças de 6 a 12 meses. Viçosa - MG.

\begin{tabular}{|c|c|c|}
\hline Variáveis & RR (IC95\%) & p valor \\
\hline \multicolumn{3}{|l|}{ Vitamina $\mathrm{A}(<\mathrm{AI})$} \\
\hline \multicolumn{3}{|l|}{ Aleitamento Materno ( $1^{\circ}$ mês) } \\
\hline AME e AMP & 1,00 & \\
\hline Misto & $1,65(1,07-2,60)$ & 0,023 \\
\hline Artificial & $1,97(1,10-3,58)$ & 0,022 \\
\hline \multicolumn{3}{|l|}{ Aleitamento Materno ( $2^{\circ}$ mês) } \\
\hline AME e AMP & 1,00 & \\
\hline Misto & $1,55(1,09-2,58)$ & 0,062 \\
\hline Artificial & $1,27(0,84-0,91)$ & 0,240 \\
\hline \multicolumn{3}{|l|}{ Aleitamento Materno ( $4^{\circ}$ mês) } \\
\hline AME e AMP & 1,00 & \\
\hline Misto & $1,16(0,65-2,05)$ & 0,615 \\
\hline Artificial & $1,06(0,62-1,83)$ & 0,821 \\
\hline Complementado & $1,27(0,80-1,99)$ & 0,302 \\
\hline \multicolumn{3}{|l|}{ Aleitamento Materno ( $6^{\circ}$ mês) } \\
\hline $\operatorname{Sim}$ & 1,00 & \\
\hline Não & $1,81(1,10-2,99)$ & 0,019 \\
\hline \multicolumn{3}{|l|}{ Ferro $(<$ EAR/AI $)$} \\
\hline \multicolumn{3}{|l|}{ Aleitamento Materno ( $1^{\circ}$ mês) } \\
\hline AME e AMP & 1,00 & \\
\hline Misto & $0,83(0,55-1,24)$ & 0,363 \\
\hline Artificial & $0,61(0,30-1,23)$ & 0,165 \\
\hline \multicolumn{3}{|l|}{ Aleitamento Materno ( $2^{\circ}$ mês) } \\
\hline AME e AMP & 1,00 & \\
\hline Misto & $0,97(0,69-1,37)$ & 0,863 \\
\hline Artificial & $1,06(0,65-1,75)$ & 0,809 \\
\hline \multicolumn{3}{|l|}{ Aleitamento Materno ( $4^{\circ}$ mês) } \\
\hline AME e AMP & 1,00 & \\
\hline Misto & $0,88(0,60-1,29)$ & 0,522 \\
\hline Artificial & $0,98(0,61-1,58)$ & 0,941 \\
\hline Complementado & $1,13(0,86-1,49)$ & 0,380 \\
\hline \multicolumn{3}{|l|}{ Aleitamento Materno ( $6^{\circ}$ mês) } \\
\hline Sim & 1,00 & \\
\hline Não & $0,71(0,49-1,05)$ & 0,095 \\
\hline
\end{tabular}

seguir as recomendações de amamentação também tenham um estilo de vida mais saudável e se atentem ao recomendado para introdução da alimentação complementar ${ }^{11,25}$.

A introdução precoce de alimentos complementares está associada ao menor aporte de nutrientes e aumento da vulnerabilidade da criança a diarreias e infecções, levando a um comprometimento do estado nutricional de vitamina $\mathrm{A}^{27}$. As crianças do presente estudo apresentaram menor consumo desse nutriente quando não amamen- tadas conforme recomendação, estando mais expostas ao risco de uma possível deficiência.

Estima-se que o gasto com fórmulas infantis para alimentação da criança corresponda a aproximadamente $35 \%$ do salário mínimo, ao passo que com leite de vaca o gasto é de $11 \%{ }^{28}$. O aleitamento materno representa uma opção mais econômica para a família e seu fornecimento à criança contribui para que uma maior parte da renda seja destinada ao gasto com outros alimentos, principalmente no que se refere aos alimen- 
tos fontes de vitamina A, como as frutas e verduras. Logo, as crianças em aleitamento materno exclusivo ou predominante tenderiam a ter uma alimentação de melhor qualidade, com maior oferta de alimentos fontes da vitamina $\mathrm{A}^{4}$.

Neste estudo observou-se a influência positiva do aleitamento materno no primeiro semestre de vida sobre o consumo alimentar das crianças no início da alimentação complementar até o fim do primeiro ano de vida. A mediana de consumo de ferro e vitamina A diferiu estatisticamente entre os grupos de aleitamento materno em todos os momentos avaliados.

O consumo de ferro foi maior após os seis meses no grupo que esteve em aleitamento artificial no primeiro mês de vida. Esse achado pode ser explicado pelo fato de que as crianças que não são amamentadas tenderem à introdução precoce da alimentação complementar, com acesso também precoce a alimentos fontes de ferro, como as carnes $^{29}$. De todo modo, é importante considerar a influência de fatores de confusão nesta análise bivariada, em que simplesmente foram comparadas as medianas entre os grupos. Quando incluídas as variáveis de confusão para ajuste, na análise multivariada, observou-se que não houve diferença na prevalência de baixa ingestão de ferro no segundo semestre de vida entre as categorias de aleitamento no primeiro semestre.

Sabe-se que a biodisponibilidade do ferro do leite materno pode reduzir em até $80 \%$ quando outros alimentos são introduzidos ${ }^{30}$. Nesse sentido, ressalta-se a importância da prática de aleitamento materno exclusivo nos primeiros seis meses de vida no que se refere à adequada ingestão deste micronutriente.

A introdução precoce de outros leites pode causar alterações na permeabilidade da mucosa intestinal do lactente, desencadeando problemas como alergias, anemia ferropriva, sobrecarga renal e deficiência de vitaminas, minerais e gorduras essenciais (ômega 3 e ômega 6) 29,31,32. Além disso, o leite materno é capaz de suprir as necessidades fisiológicas de ferro da criança quando fornecido de forma exclusiva nos primeiros seis meses, visto sua elevada biodisponibilidade quando comparado aos demais tipos de leite. Cerca de $50 \%$ do ferro ingerido através do leite materno é absorvido, enquanto que no leite de vaca há uma absorção de apenas 10\% do nutriente consumido ${ }^{30}$.
Dentre as limitações desse estudo destacamse as consideráveis perdas de seguimento, comuns em estudos de coorte. Nesse sentido, um tamanho amostral foi calculado, mostrando que houve amostra suficiente para estudar a influência do aleitamento materno no pior consumo alimentar posterior das crianças. Ademais, não houve viés diferencial quando se comparou as crianças acompanhadas com as perdidas nos primeiros seis meses. Os dados referentes à alimentação complementar foram obtidos por apenas um recordatório de $24 \mathrm{~h}$, o que impossibilitou a realização do ajuste dos nutrientes pela variabilidade intraindividual. Porém, observou-se que a alimentação das crianças nessa faixa etária foi monótona, possibilitando o uso de um único recordatório. Além disso, outros trabalhos utilizaram apenas um inquérito alimentar para avaliar a ingestão de nutrientes em crianças ${ }^{33,34}$. A junção de crianças em aleitamento materno exclusivo e predominante foi necessária devido à baixa frequência dessas em aleitamento predominante. Porém, destaca-se que os benefícios da amamentação exclusiva em relação aos outros tipos de aleitamento são indiscutíveis.

Entretanto, ressalta-se a relevância deste trabalho na investigação da relação existente entre o tipo de aleitamento e o consumo de micronutrientes no primeiro ano de vida, tendo em vista que essa associação é pouco estudada. Na prática clínica, os achados deste estudo reforçam a importância do aleitamento materno na formação de hábitos alimentares mais saudáveis, contribuindo também para a prevenção de doenças associadas à má alimentação na infância. Ou seja, além dos inúmeros benefícios da amamentação à saúde da criança ${ }^{3}$, essa prática influencia positivamente as preferências alimentares em fases posteriores ${ }^{13}$.

Em conclusão, mais de um terço das crianças tinham consumo abaixo da recomendação para vitamina A e ferro. As crianças que amamentaram de maneira exclusiva ou predominante no primeiro semestre de vida apresentaram maior frequência de ingestão adequada de vitamina A. $O$ consumo de ferro foi menor entre as crianças amamentadas, porém, a biodisponibilidade desse nutriente no leite materno é maior. Esses achados reforçam a importância de políticas públicas com o grupo materno-infantil em prol do incentivo ao aleitamento materno exclusivo e à introdução correta da alimentação complementar. 


\section{Colaboradores}

MA Silva: coleta e análise dos dados, elaboração do artigo. MM Soares: coleta e análise dos dados, elaboração do artigo. PCA Fonseca: coleta e análise dos dados, elaboração do artigo. SA Vieira: coleta e análise dos dados, elaboração do artigo. CA Carvalho: coleta e análise dos dados, elaboração do artigo. RM Amaral: análise dos dados e elaboração do artigo. SCC Franceschini: elaboração do artigo e orientação do trabalho. JF Novaes: elaboração do artigo e orientação do trabalho.

\section{Agradecimentos}

À Fundação de Amparo à Pesquisa do Estado de Minas Gerais - Fapemig. 


\section{Referências}

1. Lima APE, Javorski M, Amorim RJM, Oliveira SC, Vasconcelos MGL. Práticas alimentares no primeiro ano de vida: representações sociais de mães adolescentes. Rev Bras Enferm 2014;67(6):965-971.

2. World Health Organization (WHO). Report of an expert consultation on the optimal duration of exclusive breastfeeding. Geneva: WHO; 2001.

3. Brasil. Ministério da Saúde (MS). Secretaria de Atenção à Saúde. Departamento de Atenção Básica. Saúde da criança: aleitamento materno e alimentação complementar. 2a Edição. Brasília: MS; 2015.

4. Brasil. Ministério da Saúde. Secretaria de Atenção à Saúde. Departamento de Atenção Básica. Saúde da criança: nutrição infantil: aleitamento materno e alimentação complementar. Brasília: MS; 2009.

5. Dias CAP, Freire LMS, Franceschini SCC. Recomendações para alimentação complementar de crianças menores de dois anos. Rev. Nutr 2010; 23(3):475-486.

6. Alves CRL, Santos LC, Goulart LMHF, Castro PR. Alimentação complementar em crianças no segundo ano de vida. Rev. Paul. Pediatr 2012;30(4):499-506.

7. Mariath AB, Giachini RM, Lauda LG, Grillo LP. Estado de ferro e retinol sérico entre crianças e adolescentes atendidos por equipe da Estratégia Saúde da Família de Itajá, Santa Catarina. Ciên Saúde Colet 2010;15(2):509-516

8. Silva MA, Carvalho CA, Fonsêca PCA, Vieira AS, Ribeiro AQ, Priore SE, Franceschini ACC. Prevalência e fatores associados à anemia ferropriva e hipovitaminose A em crianças menores de um ano. Cad Saude Colet 2015;23(4):362-367.

9. Brasil. Ministério da Saúde (MS). Pesquisa Nacional de Demografia e Saúde da Criança e da Mulher (PNDS) 2006: dimensões do processo reprodutivo e da saúde da criança [livro na Internet]. Brasília: MS; 2009 [acessado 2016 Mai]. Disponível em: http://bvsms.saude.gov. br/bvs/publicacoes/pnds_crianca_mulher.pdf

10. Burnier D, Dubois L, Girard M. Exclusive breastfeeding duration and later intake of vegetables in preschool children. Eur J Clin Nutr 2011;65(2):196-202.

11. Soldateli B, Vigo A, Giugliani ERJ. Effect of pattern and duration of breastfeeding on the consumption of fruits and vegetables among preschool children. PloS One 2016;11(2):1-8.

12. Lauzon-Guillain B, Jones L, Oliveira A, Moschonis G, Betoko A, Lopes C, Moreira P, Manios Y, Papadopoulos NG, Emmett P, Charles MA. The influence of early feeding practices on fruit and vegetable intake among preschool children in 4 European birth cohorts. Am J Clin Nutr 2013;98:1-9.

13. Perrine CG, Galuska DA, Thompson MPH, Scanlon KS. Breastfeeding Duration Is Associated With Child Diet at 6 Years. Pediatrics 2014;134(1):850-855.

14. Valmórbida JL, Vitolo MR. Factors associated with low consumption of fruits and vegetables by preschoolers of low socio-economic level. J Pediatr (Rio J) 2014;90(5):464-471.

15. Fonseca PCA, Carvalho CA, Ribeiro SAV, Nobre LN, Pessoa MC, Ribeiro AQ, Priore SE, Franceschini SDCC. Determinants of the mean growth rate of children under the age of six months: a cohort study. Ciên Saude Colet 2016;22(8):2713-2726.
16. Carvalho CA, Ribeiro SAV, Nobre LN, Pessoa MC, Ribeiro AQ, Priore SE, Franceschini SCC. Fatores sociodemográficos, perinatais e comportamentais associados ao tipo de leite consumidos por crianças menores de seis meses: coorte de nascimento. Ciên Saude Colet 2017;22(8):2713-2726.

17. Tabela Brasileira de Composição de Alimentos (TACO). 4a Edição revisada e ampliada. Campinas, SP: UNICAMP; 2011. Disponível em: http://www. unicamp.br/nepa/taco/contar/taco_4_edicao_ampliada_e_revisada.pdf?arquivo=ta co_4_versao_ampliada_e_revisada.pdf

18. Institute Of Medicine/Food And Nutrition Board. Dietary References Intakes for vitamin A, vitamin $K$ arsenic, boron, chromium, copper, iodine, iron, manganese, molybdenum, nickel, silicon, vanadium and zinc. Washington: National Academy Press; 2001.

19. Willett W. Nutritional epidemiology. 2a Edição. New York: Oxford; 1998.

20. Slater B, Marchioni DML, Voci SM. Aplicação de regressão linear para correção de dados dietéticos. Rev Saude Publica 2007;41(2):190-196.

21. World Health Organization (WHO). United Children's Fund (WHO/UNICEF). Consultation on complementary feeding in Montpellier. Subsídios preprados pela WHO e UNICEF. Montpellier: WHO; 1995.

22. Fidelis CMF, Osório MM. Consumo alimentar de macro e micronutrientes de crianças menores de cinco anos no Estado de Pernambuco, Brasil. Rev Bras Saude Matern Infant 2007;7(1):63-74.

23. Freiberg CK, Philippi ST, Leal GVS, Martinez MF, Silva MEW. Avaliação do consumo alimentar de crianças menores de dois anos institucionalizadas em creches no município de São Paulo. Rev Assoc Bras Nutr 2012;4(5):17-21.

24. Kurihayashi AY, Augusto RA, Escaldelai FMD, Martini LA. Estado nutricional de vitaminas A e D em crianças participantes de programa de suplementação alimentar. Cad Saude Publica 2015;31(3):531-542.

25. Moller LM, Hoog ML, van Eijsden M, Gemke RJ, Vrijkotte TG. Infant nutrition in relation to eating behaviour and fruit and vegetable intake at age 5 years. $\mathrm{Br}$ J Nutr 2013;109:564-571.

26. World Health Organization (WHO). Global prevalence of vitamin A deficiency in populations at risk 19952005: WHO global database on vitamin A deficiency. Geneva: WHO; 2009.

27. Araújo MFM, Fiaco AD, Pimentel LS, Schmitz BAS. Custo e economia da prática do aleitamento materno para a família. Rev Bras Saude Matern Infant 2004;4(2):135-141.

28. Schincaglia RM, Oliveira AC, Sousa LM, Martins KA. Práticas alimentares e fatores associados à introdução precoce da alimentação complementar entre crianças menores de seis meses na região noroeste de Goiânia. Epidemiol Serv Saude 2015;24(3):465-474.

29. Bortolini GA, Vitolo MR. Importância das práticas alimentares no primeiro ano de vida na prevenção da deficiência de ferro. Rev Nutr 2010;23(6):1051-1062.

30. Ferreira S, Pinto M, Carvalho P, Gonçalves JP, Lima $\mathrm{R}$, Pereira $\mathrm{F}$. Alergia às proteínas do leite de vaca com manifestações gastrointestinais. Revista de Pediatria do Centro Hospitalar do Porto 2014;23(2):72-79. 
31. Machado AKF, Elert VW, Pretto AD, Pastore CA. Intenção de amamentar e de introdução de alimentação complementar de puérperas de um Hospital Escola do Sul do Brasil. Ciên Saude Colet 2014;19(7) 19831989.

32. Costa EC, Silva SP, Lucena JR, Batista Filho M, Lira PI Ribeiro MA, Osório MM. Food consumption of children from cities with a low human development index in the Brazilian Northeast. Rev Nutr 2011;24:395-405.

33. Silva JV, Timóteo AK, Santos CD, Fontes G, Rocha EM. Food consumption of children and adolescents living in an area of invasion in Maceio, Alagoas, Brazil. Rev Bras Epidemiol 2010;13:83-93.

34. Menezes RCE, Osório MM. Consumo energético -proteico e estado nutricional de crianças menores de cinco anos, no estado de Pernambuco, Brasil. Revista de Nutrição 2007;20(4):337-347.

Artigo apresentado em 23/03/2017

Aprovado em 24/04/2018

Versão final apresentada em 26/04/2018 